\title{
Computational Procedure to an accurate DFT simulation to solid state systems
}

Eduardo O. Gomes ${ }^{1,4}$, Guilherme S. L. Fabris ${ }^{2}$, Mateus M. Ferrer ${ }^{3}$, Fabiana V. Motta ${ }^{1}$, Mauricio R. D. Bomio ${ }^{1}$, Juan Andres ${ }^{4}$,Elson Longo ${ }^{5}$ and Julio R. Sambrano ${ }^{2 *}$

${ }^{1}$ LSQM - Laboratory of Chemical Synthesis of Materials, Federal University of Rio Grande do Norte, Natal, RN, Brazil.

${ }^{2}$ Modeling and Molecular Simulation Group - CDMF, São Paulo State University, 17033-360, Bauru, SP, Brazil.

${ }^{3}$ Department of Physics, Federal University of Pelotas, 96010-610, Pelotas, RS, Brazil

${ }^{4}$ Department of Analytical and Physical Chemistry, University Jaume I (UJI), Castelló 12071, Spain.

${ }^{5}$ Chemistry Institute - CDMF, Federal University of São Carlos, P.O. Box 14801-907, São Carlos, SP, Brazil.

KEYWORDS: Quantum Computation Methodology, DFT, Basis set optimization, $\mathrm{BaMoO}_{4}$ 


\section{ABSTRACT}

The density functional theory has become increasingly common as a methodology to explain the properties of crystalline materials because of the improvement in computational infrastructure and software development to perform such computational simulations. Although several studies have shown that the characteristics of certain classes of materials can be represented with great precision, it is still necessary to improve the methods and strategies in order to achieve more realistic computational modeling. In the present work, strategies are reported in a systematic way for the accurate representation of crystalline systems. The crystalline compound chosen for the study as a case test was $\mathrm{BaMoO}_{4}$, both because of its potential technological application and because of the low accuracy of the simulations previously reported in the literature. The computational models were carried out with the B3LYP and WC1LYP functionals selected from an initial set containing eight hybrid functionals in conjunction with an all-electron basis set. Two different strategies were applied for improving the description of the initial models, both involving atomic basis set optimization and Hartree-Fock exchange percentage adjustment. The results obtained with the two strategies show a precision of structural parameters, band gap energy, and vibrational properties never before presented in theoretical studies of $\mathrm{BaMoO}_{4}$. Finally, a flowchart of good calculation practices is elaborated. This can be of great value for the organization and conduction of calculations in new research. 


\section{INTRODUCTION}

In theoretical approaches, the elaboration of computational models that represent the reality is still a challenge, especially for material science. ${ }^{1}$ However, the search for models with an accurate approximation of structural, vibrational, and electronic characteristics is very useful for trends, elucidations, and even in findings of new observable characteristics and properties., ${ }^{2,3}$

The solid-state simulation still presents a set of hindrances for the correct description of the electronic structure by purely ab initio methods. As much as the approximation is more and more accurate, the calculations strategies constantly confront the results with experimental parameters, such as band gap energy, vibrational modes, and other target properties, in order to orientate the adopted parameters of the theoretical methods. The constant existence of distortions and stacking faults in real crystals can also generate structural characteristics very different from the expected theoretical global minimum.

The first generalizations of the Hartree-Fock (HF) equations adapted to crystalline systems were performed in the late $1960 \mathrm{~s}^{4,5}$ and, only in the early $1970 \mathrm{~s}$, the first ab initio calculation for a crystalline system (diamond) was performed, with good accuracy in determining the structural parameters. ${ }^{6}$ After several years and with the parallel development of computing science, it was possible to obtain many properties for crystalline solid by $a b$ initio methods: structural optimization, vibrational harmonic frequencies at $\Gamma$ point (infrared and Raman) and phonon dispersion, thermodynamic properties, reflectance spectra, study of solid solutions, point defects, excited states, band structure, total and projected density of state, charge density and electrostatic potential maps, and magnetic and dielectric properties, among others.

Much of the success of these advances are attributed to the density functional theory (DFT), ${ }^{7,8}$ whose basic idea is that the electron density of the system determines the ground state properties. 
Nowadays, DFT methods are the first choice for the computational treatment of solids, providing results comparable to multiconfigurational methods with a considerably low computational cost.

Usually, the choice of the density functional, that includes the exchange-correlation functional, and the use of an appropriate atomic basis set is the first procedure in DFT simulations. An appropriate choice can lead to accurate target properties being simulated reliably, especially when dealing with models for which there are no experimental information for comparison. ${ }^{9}$ In particular, hybrid functional has been employed with success to simulate solid-state systems, such as the study of the bulk, ${ }^{10,11}$ surfaces, ${ }^{12}$ nanotubes ${ }^{13}$, adsorption, and doping processes. ${ }^{14}$ As a popular example, the $\mathrm{B} 3 \mathrm{LYP} \mathrm{P}^{15-17}$ hybrid functional is a common choice to describe the properties of several systems.

As we know, the exact exchange-correlation functional is unknown; therefore, a considerable amount of research has been carried out in the past decades to find accurate approximations for the exact functional. There is no unique method to find these approximations, and, among these, the introduction and adjustments of empirical parameters are the most used methodology. According to Bredow and Gerson, ${ }^{18}$ the optimal percentage of each functional term can change depending on the system and may also be the target of study for a better approximation of the results.

When considering the atomic basis set, there are several available bases of each atom described in the literature that can be used for molecular or crystalline systems in order to expedite the study of materials. However, it is important to highlight that the development of a specific basis set for a molecule cannot always be successfully applied to solid-state systems, or a basis generated for a specific solid-state system can be appropriately used for others. Mostly, it 
is necessary to generate a new basis or adjust a predefined basis set, such as the optimization of the coefficients and/or exponents of the most external or more specific shells.

Based on that the above, the aim of this study was to investigate two different strategies to conceive computational models, taking as an example the particular case of the metallic molybdate, $\mathrm{BaMoO}_{4}(\mathrm{BMO})$. Both strategies consider the optimization of the atomic basis set and new parameterization of the mixing fraction of the HF exchange functional. Structural, electronic, and vibrational property data obtained by means of both strategies were compared with experimental and theoretical data reported in the literature to compare and evaluate their accuracy

\section{PROBE SYSTEM}

To verify the efficiency of the proposed strategy, it was chosen a system belonging to an important family of inorganic materials $\left(\mathrm{AMoO}_{4}\right)$ that have a high potential for application in a great diversity of technological devices. ${ }^{19-21}$ Under typical conditions, when solids of this class are composed of relatively large bivalent cations $(\mathrm{A}=\mathrm{Ba}, \mathrm{Ca}, \mathrm{Sr}$, and $\mathrm{Pb})$, they assume a scheelite-type tetragonal structure. ${ }^{22}$

In particular, (BMO) is cited as one of the most important members of this family, mainly because of its photoluminescent properties. ${ }^{23-25}$ However, a considerable number of studies have reported several potential applications of BMO, such as its uses as anodes in lithium and sodium batteries, ${ }^{26}$ catalyzers, ${ }^{27}$ component in white $\operatorname{LEDS}^{28}$, and thermoelectric devices. ${ }^{29}$ This versatility is the result of the structural and shape modifications of its nanoparticles, ${ }^{30,31}$ as a function of the synthesis methodology, such as sonochemical, ${ }^{32}$ electrochemical,${ }^{33}$ microwaveassisted citrate complex, ${ }^{34}$ and microwave-assisted hydrothermal. ${ }^{27}$ 
Looking for theoretical studies on BMO, it was noticed that there have been few reported in this field. Among these, Zhao et al. applied the DFT and fully relativistic self-consistent DiracSlater theory to explore the behavior of interstitial oxygen around the $\mathrm{Mo}^{6+}$ ions on the structure. They concluded that there was an interstitial oxygen combined with the nearest-neighbor lattice oxygen ions to form molecular ions $O_{3}^{4-}$ and $O_{4}^{4-} \cdot{ }^{35}$ Qin et al. used DFT combined with PBE functional to give support to experimental results about the dielectric improvement as a function

of pressure. ${ }^{36}$ Sczancoski and coworkers reported a combined experimental and DFT study with B3LYP hybrid functional, to explain the optical properties of $\mathrm{BaMoO}_{4}$ obtained by a coprecipitation method in the presence of polyethylene glycol. ${ }^{37}$

\section{COMPUTATIONAL SETUP AND MODELS SYSTEM}

The structural and electronic properties of BMO bulk were simulated by means of periodic DFT using the CRYSTAL17 package. ${ }^{38}$

The BMO has a tetragonal unit cell that belongs to the space group I41/a (symmetry $\left.\mathrm{C}_{4 \mathrm{~h}}^{6}\right)^{37}$ with a band gap energy of $\sim 4.54 \mathrm{eV}^{39}$ and experimental lattice parameters $a=b=5.59 \AA$ and $c=$ $12.82 \AA$, as in Figure $1 .^{40}$ 


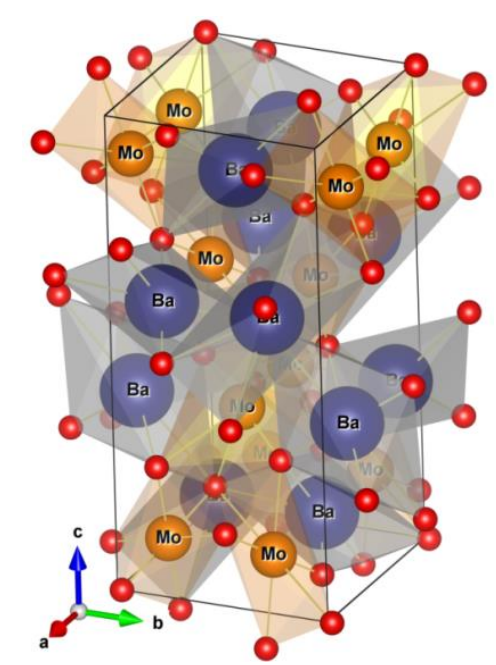

Figure 1. Unit cell of the BaMoO4: the Oxygen atoms are represented by the red spheres.

A set of the most-used functionals in solid-state simulations was initially tested: $\mathrm{B} 1 \mathrm{WC},{ }^{41}$ B3PW, ${ }^{15}$ PBE0,${ }^{42}$ PBESOL0, ${ }^{43}$ SOGGAXC,${ }^{44}$ HSE06, ${ }^{45}$ WC1LYP,${ }^{46}$ and B3LYP. ${ }^{15-17}$

To choose the starting basis set combination, from the B3LYP functional, several atomic basis sets (all-electron and pseudopotentials), available in the CRYSTAL program and Mike Towler basis set library (http://www.crystal.unito.it/basis-sets.php and http://www.tcm.phy.cam.ac.uk/ mdt26/basis_sets/Ba_basis.txt, respectively) were tested, whose calculated structural parameters and band gap energy were compared with experimental data. The chosen barium atomic center (all-electron basis set), described in Mike Towler library, presented good results for the description of our model. The selected molybdenum and oxygen all-electron basis atom centers were 976-311(d631)G, and 6-31G*, respectively. For a more detailed description about these basis sets, refer to the supplementary material.

Most of the available atomic basis sets are pre-optimized for a specific system that includes different structures and atomic interactions. This means an optimization process may be necessary to adjust, at the same time, all selected atomic bases to a target system. In this work, Powell's algorithm method ${ }^{47}$, combined with the CRYSTAL program, was used to perform the 
basis set optimization of the exponent of the valence shell functions to an energy convergence of $10^{-6}$ Hartree.

The level of accuracy on the convergence criteria for bi-electronic integrals was controlled by a set of five thresholds $\left(10^{-7}, 10^{-7}, 10^{-7}, 10^{-7}\right.$, and $\left.10^{-14}\right)$. These parameters represent the overlap and penetration for Coulomb integrals, the overlap for HF exchange integrals, and the pseudooverlap, respectively. The shrinking factor (Pack-Monkhorst and Gilat net) was set to 6, corresponding to 36-k-points in the irreducible Brillouin zone.

All stationary points were characterized as a minimum by diagonalizing the Hessian matrix with respect to atomic coordinates and unit cell parameters. The vibrational modes at the $\Gamma$ point were evaluated using the numerical second derivatives of the total energies estimated with the coupled perturbed HF/Kohn-Sham algorithm. ${ }^{48,49}$ The convergence was checked on gradient components and nuclear displacements with tolerances on their root mean square set to 0.0001 and 0.0004 a.u., respectively. The band structure and density of states (DOS) were analyzed with the same k-point sampling employed for the diagonalization of the Fock matrix in the optimization process.

\section{RESULTS AND DISCUSSION}

\subsection{Choice of functional, basis set, and strategies of calculations}

As a starting point, the optimization of the structural parameters and band gap energy calculations was performed using the selected atomic basis and different hybrid functionals, as described in Table S1 (supplementary material) and shown in Figure 2. 


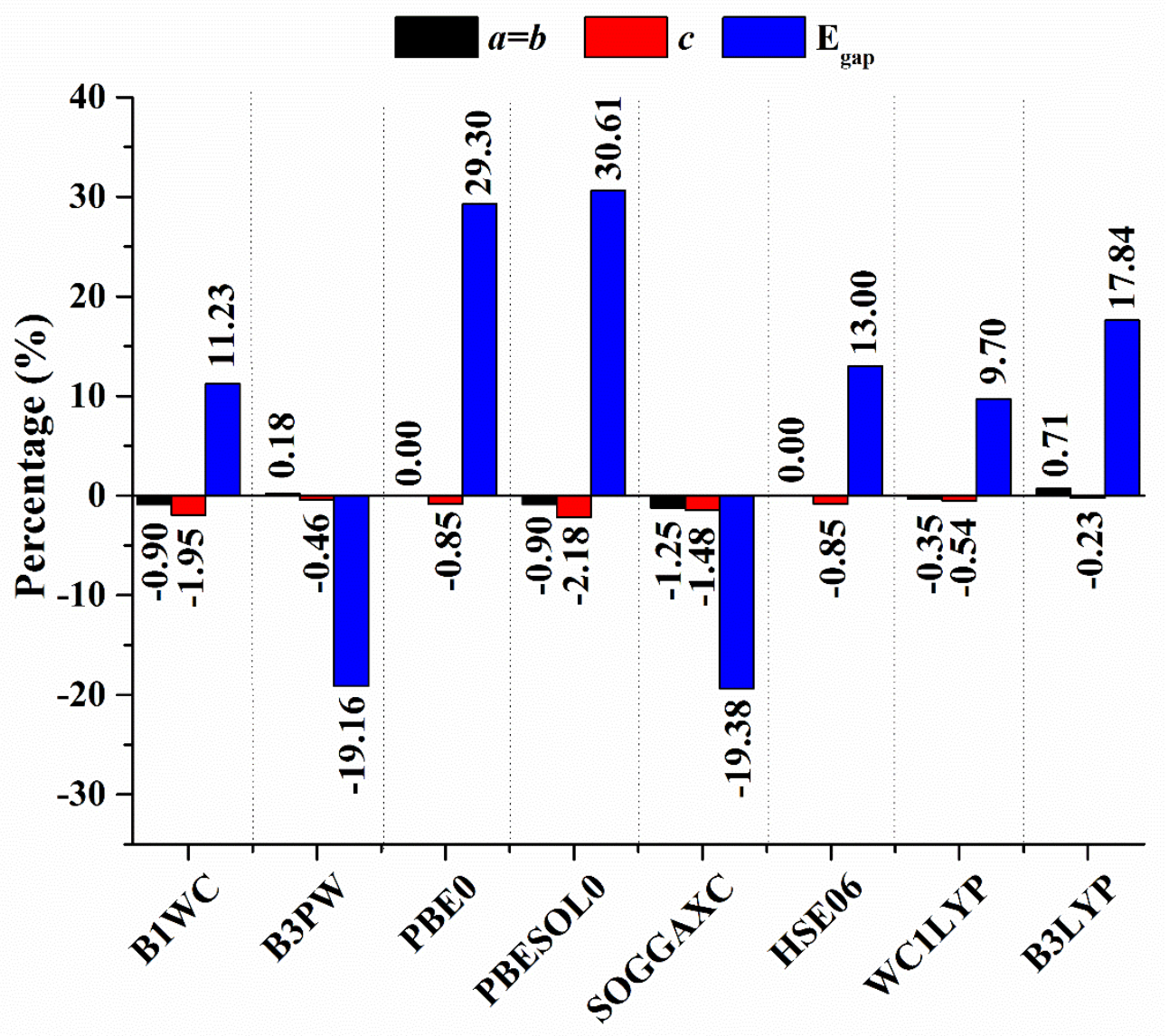

Figure 2. Calculated percentage difference for the lattice parameters and band gap energy with the original basis set for all tested hybrid functional. The experimental lattice parameters are defined as the reference.

It was observed that the WC1LYP functional describes the structural parameters $(a=b$ and $c)$ and band gap energy with the best average accuracy to the reference experimental system. The HSE06, B1WC, and B3LYP functionals also showed good and acceptable structural parameters but with discrepant band gap energy.

Nevertheless, the B3LYP functional was also used to perform the simulations for the sake of comparison with the WC1LYP, because it is one of the most used functional to study crystalline materials. ${ }^{50}$ Although the results have been somewhat favorable, they can still be improved. 
Two different strategies were used with the last two selected functionals to obtain more precise results. The first consisted of the optimization of the basis set as a function of the total energy, changing the coefficients of most external orbitals of Ba ( $s p$ and $d$ shells), Mo ( $s p$ and $d$ shells), and $\mathrm{O}(s p$ shell), and fixing the experimental lattice parameters. After updating with the new basis set coefficients, the next step was the optimization of the lattice parameters, whose results were compared again with the experimental data. This choice is arbitrary, other coefficients and exponents of another orbital could be chosen for optimization. It is usually necessary to observe the accuracy of the results as a function of the optimized coefficients. If this procedure is not satisfactory, can be added new coefficients in the optimization process.

After this procedure, the possibility of improvement of the results was verified by means of a new structural optimization, changing the percentage of the HF exchange parameter (HF\%).

The B3LYP functional is described by $E_{x c}=(1-A) \cdot\left(E_{x}^{L D A}+0.9 \cdot E_{x}^{B E C K E}\right)+A \cdot E_{x}^{H F}+$ $0.19 \cdot E_{c}^{L D A}+0.81 \cdot E_{c}^{G G A}$, where $A$ is the hybrid percentage $(\mathrm{A}=0.20$ for B3LYP) and, for the WC1LYP functional, is defined by $E_{x c}=(1-A) \cdot E_{x}^{W C}+A \cdot E_{x}^{H F}+E_{c}^{L D A}+E_{c}^{G G A}$ where $\mathrm{A}=$ 0.16 , which involves mixing the nonlocal HF exchange WC with the LYP correlation.

The second strategy was the inverse of the first procedure. Structural optimization was performed in which the influence of the HF\% variation was analyzed, and, subsequently, the atomic basis set was optimized (same most external orbitals of each atomic center) with the new hybrid functional that considered the new HF\%.

The preliminary structural and electronic results of both paths are described below.

\subsubsection{First path: basis set optimization followed by HF\% analysis (BasisOpt/ HF\%)}


The valence shell values of the optimized basis with standard WC1LYP and B3LYP functional can be found in Table 1.

Table 1. New coefficients of the optimized basis set.

\begin{tabular}{ccccc}
\hline Atom & Orbital & Original & WC1LYP & B3LYP \\
\hline $\mathrm{Ba}$ & ${ }^{1} \alpha_{s p}$ & 0.4539 & 0.207828443199 & 0.206427618918 \\
$\mathrm{Ba}$ & $\alpha_{d}$ & 0.572 & 0.524003777186 & 0.521591022655 \\
$\mathrm{Ba}$ & ${ }^{2} \alpha_{s p}$ & 0.5286 & 0.528469224165 & 0.521508521757 \\
$\mathrm{Mo}$ & ${ }^{1} \alpha_{s p}$ & 0.3534 & 0.332025568342 & 0.334604789514 \\
$\mathrm{Mo}$ & $\alpha_{d}$ & 0.1781 & 0.183156130810 & 0.179441331338 \\
$\mathrm{Mo}$ & ${ }^{2} \alpha_{s p}$ & 0.1424 & 0.090437621023 & 0.102334203450 \\
$\mathrm{O}$ & $\alpha_{s p}$ & 0.2742 & 0.250441193509 & 0.253500560333
\end{tabular}

The index ${ }^{1}$ and ${ }^{2}$ refer to the position of coefficient in the respective basis set.

The calculated lattice parameters and band gap energy after this procedure were $a=5.62$ (5.68) $\AA, c=12.76(12.91) \AA$, and $4.81(5.20) \mathrm{eV}$ to the WC1LYP (B3LYP). The basis set optimization changed the structural parameters ( $a$ and $c$ ) to slightly worse values in comparison with the experimental ones (last row of Table S1). However, there were improvements in band gap energy on both functionals, with a correction of $3 \%$. An interesting point to highlight in Table 1 is that the coefficients ${ }^{1} \alpha_{s p}$ of $\mathrm{Ba}$ and ${ }^{2} \alpha_{s p}$ of Mo atoms are the ones that had the greatest value variation.

These new outputs were then used to elaborate the starting point of the gradual modification of the HF\% of the WC1LYP and B3LYP functionals. The structural parameters and band gap energy of each HF\% modulation are shown in Figure 3 and Table S2. These results indicated a 
better description with $12 \%$ of $\mathrm{HF}$ contribution, in relation to the experimental characteristic previously described.

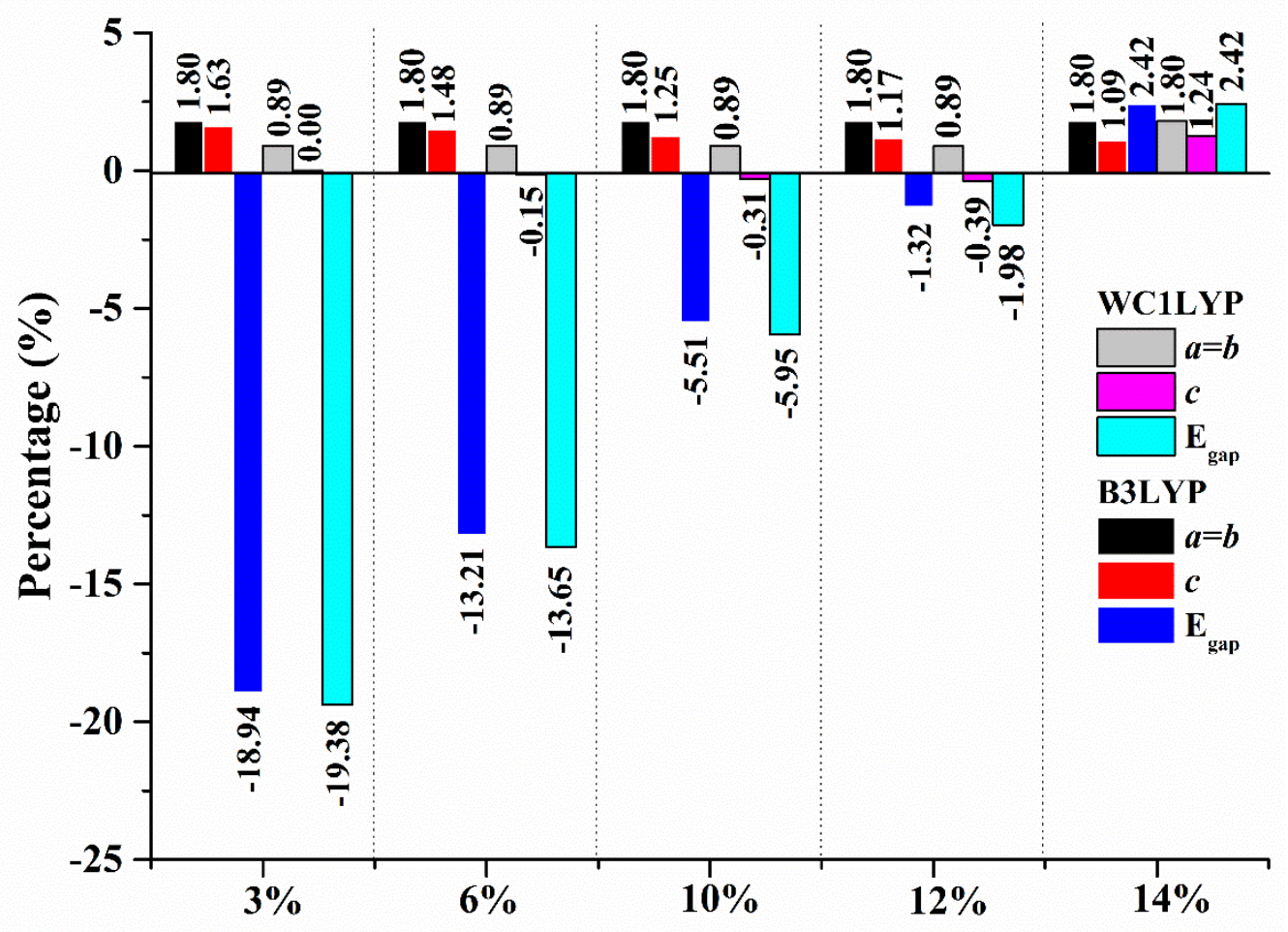

Figure 3. Calculated percentage difference for the lattice parameters and band gap energy with the optimized basis set following the first path for five different values of $\mathrm{HF} \%$ with the WC1LYP and B3LYP functionals. The experimental lattice parameters are defined as the reference.

The results of $12 \%$ of $\mathrm{HF} \%$ are very close to the experimental values for both functionals. However, the B3LYP represented more closely the experimental band gap energy (difference of $1.32 \%$ ) while the WC1LYP resulted in better values of the structural parameters in the final calculations (difference of $a=0.89 \%$ and $c=0.39 \%$ ). Even though the B3LYP values started from more discrepant results, after all these processes, both functionals presented acceptable final results for the structural parameters and band gap values. 


\subsubsection{Second path: HF\% analysis followed by basis set optimization (HF\%/BasisOpt)}

Figure 4 and Table S3 depict the structural parameters and band gap values of all tested HF\% using the original atomic basis.

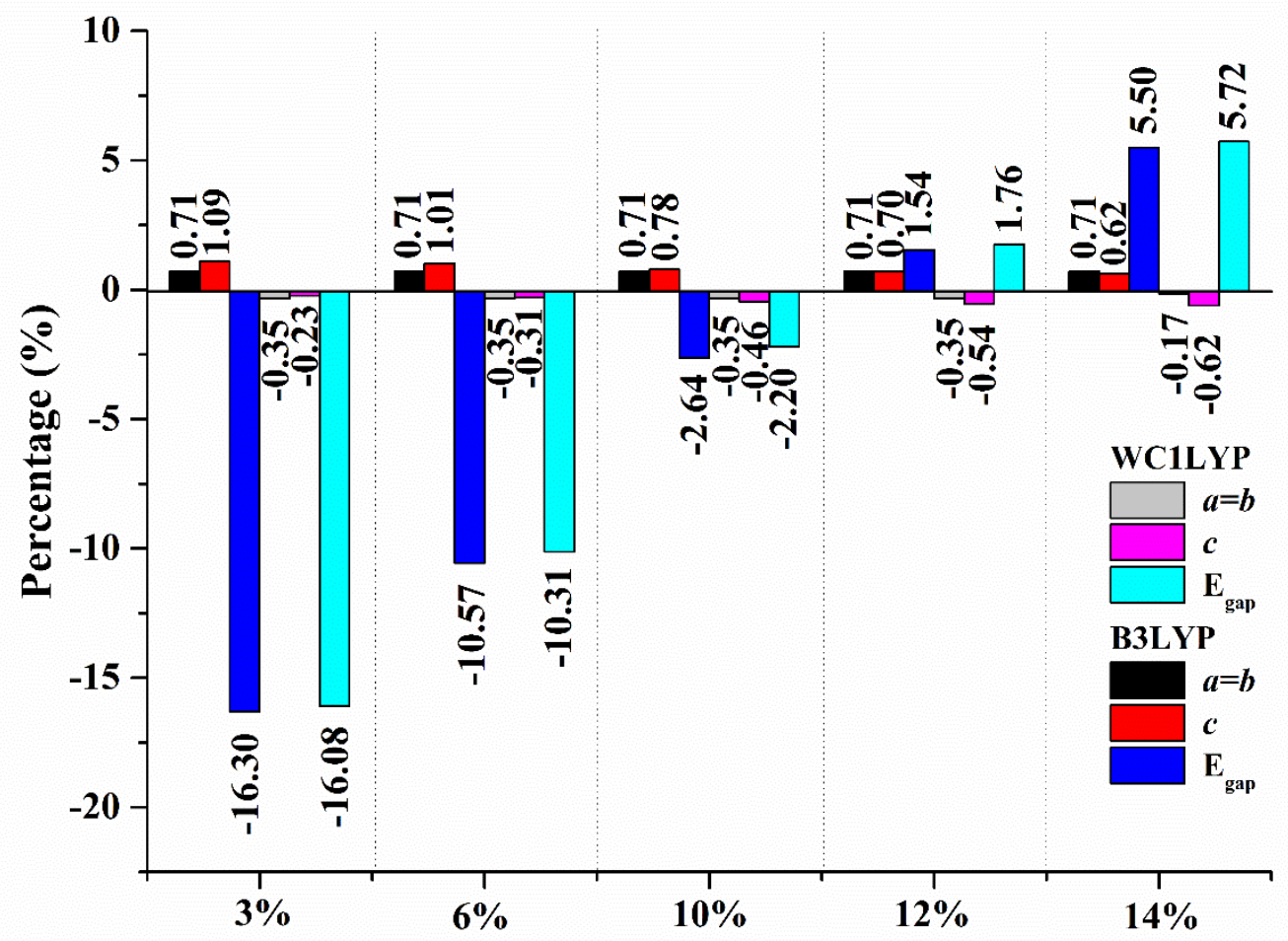

Figure 4. Calculated percentage difference for the lattice parameters and band gap energy with the original basis set following the second path for five different values of $\mathrm{HF} \%$ with the WC1LYP and B3LYP functionals. The experimental lattice parameters are defined as the reference.

The values depicted in Figure 4 show that the $12 \%$ percentage had better proximity to the experimental results and, therefore, was selected to be used in the atomic basis optimization. 
The valence shell values of the optimized atomic basis with modified WC1LYP(12\%) and B3LYP(12\%) can be found in Table 2.

Table 2. New coefficients of the optimized basis set.

\begin{tabular}{ccccc}
\hline Atom & Orbital & Original & WC1LYP(12\%) & B3LYP(12\%) \\
\hline $\mathrm{Ba}$ & $\alpha_{d}$ & 0.572 & 0.528864378892 & 0.524309031231 \\
$\mathrm{Ba}$ & ${ }^{1} \alpha_{s p}$ & 0.5286 & 0.207786429464 & 0.20819367716 \\
$\mathrm{Ba}$ & ${ }^{2} \alpha_{s p}$ & 0.4539 & 0.522602907967 & 0.51940215471 \\
$\mathrm{Mo}$ & ${ }^{1} \alpha_{s p}$ & 0.3534 & 0.331036558642 & 0.332938970171 \\
$\mathrm{Mo}$ & ${ }^{2} \alpha_{s p}$ & 0.1424 & 0.090437634754 & 0.101253952411 \\
$\mathrm{Mo}$ & $\alpha_{d}$ & 0.1781 & 0.183070751205 & 0.182764137322 \\
$\mathrm{O}$ & $\alpha_{s p}$ & 0.2742 & 0.249504780874 & 0.251423714332
\end{tabular}

The index ${ }^{1}$ and ${ }^{2}$ refer to the position of coefficient in the respective basis set.

The calculations, considering the new coefficients, resulted in structure parameters of $a=b=$ 5.64 (5.69) $\AA, c=12.77$ (12.97) $\AA$, and 4.45 (4.46) $\mathrm{eV}$ for the WC1LYP(12\%) (B3LYP(12\%)) functional. It is also worth to comment that, similarly to what happens with the coefficients on the first path, the ${ }^{1} \alpha_{s p}$ of $\mathrm{Ba}$ and ${ }^{2} \alpha_{s p}$ of Mo atoms are the ones that had the greatest value variation.

The values obtained of the structural parameters and band gap energy values showed that both simulation paths can provide accurately similar results as compared with the experimental data. The use of the BasisOpt/ HF\% path resulted in lower band gap energy $(\sim 0.1$ below the experimental value), and the $\mathrm{HF} \%$ /BasisOpt path showed an opposite characteristic, with higher band gap values $(\sim 0.1$ above the experimental value). The use of the B3LYP functional, even 
with initial discrepant results, showed great improvement by means of the proposed routes. The computational model represents an ideal structure, free of distortions or potential defects, and the structural parameters and band gap energy values of the experimental measurements have errors of precision of the used devices and methods of analysis.

\subsection{Electronic properties}

Each one of the final four models was well explored to understand the consequences of the calculation route choices. A better description of electronic characteristics was performed by evaluating the band structure and DOS, depicted in Figures 5 and 6, respectively.

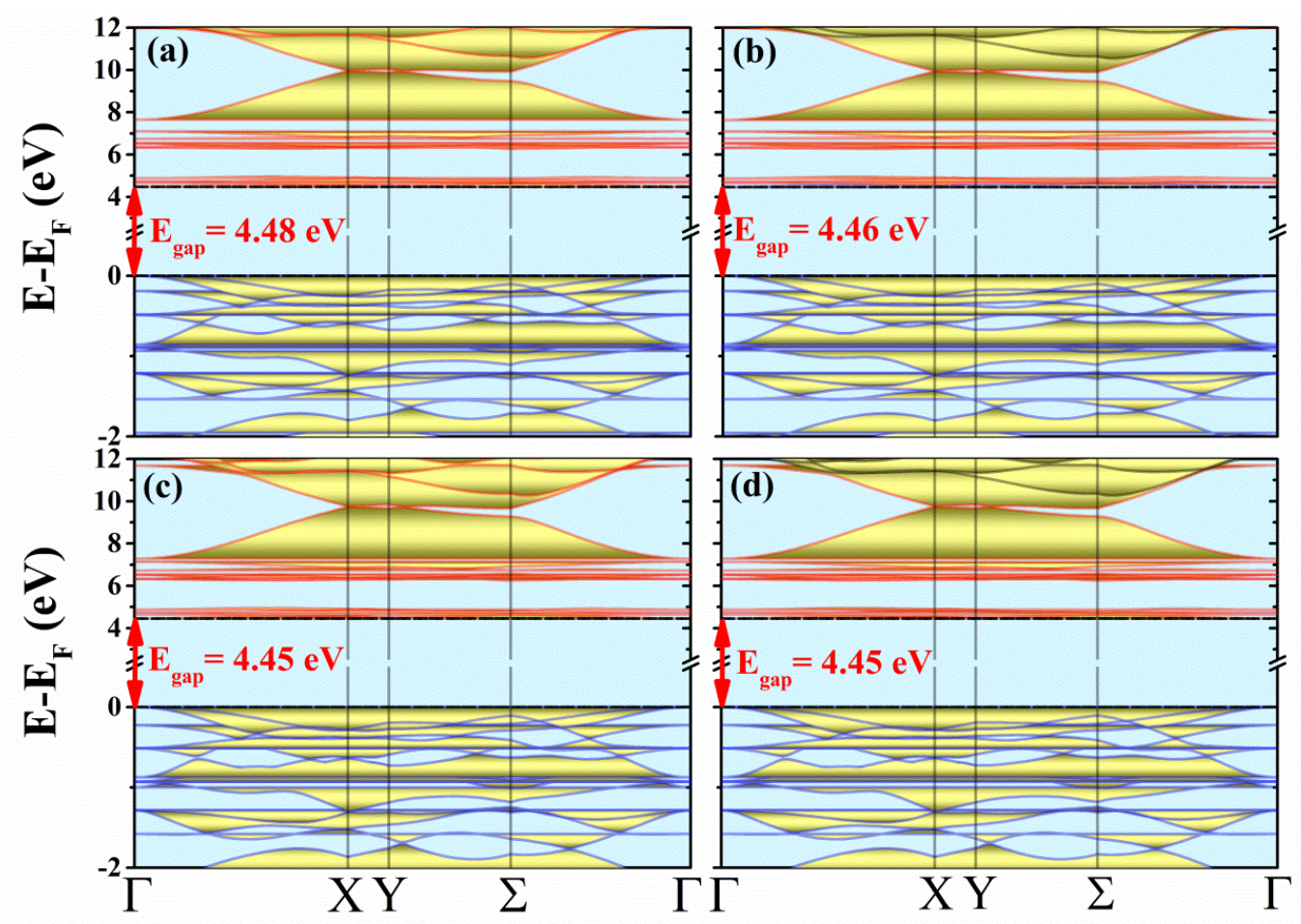

Figure 5. Band structure of the BMO in the case of: (a) B3LYP with basis set optimizations followed by the HF\% modification, (b) B3LYP with HF\% modification followed by basis set 
optimizations, (c) WC1LYP with basis set optimizations followed by the HF\% modification, and (d) WC1LYP with HF\% modification followed by basis set optimizations.

As can be observed in Figure 5, the band structure profiles of all models are very similar. In addition, the top of the valence band and the bottom of the conduction band are located at the $\Gamma$ point, which indicates a direct band gap for the four models.

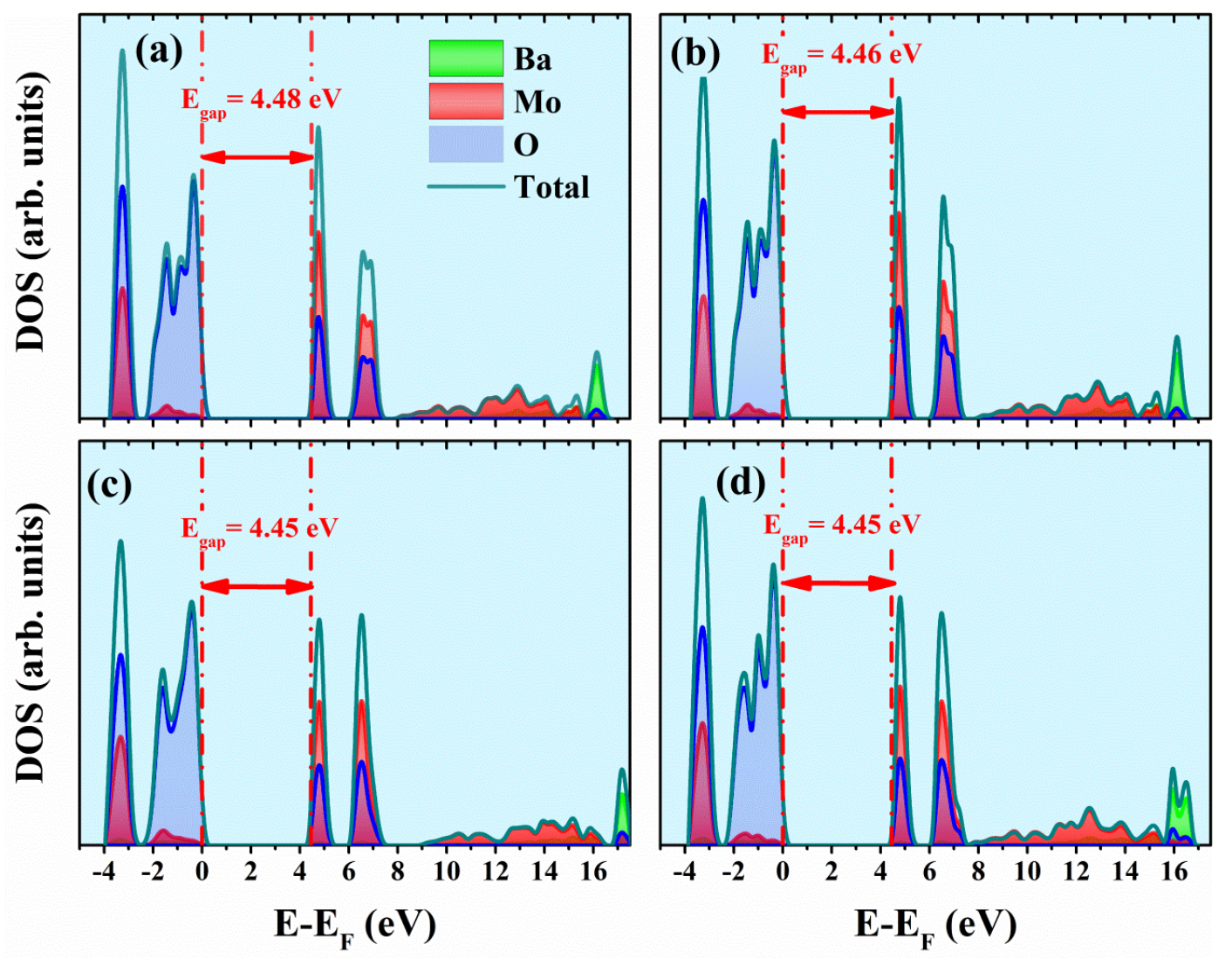

Figure 6. Density of states of the $\mathrm{BaMoO}_{4}$ in the case of: (a) B3LYP with basis set optimizations followed by the HF\% modification, (b) B3LYP with HF\% modification followed by basis set optimizations, (c) WC1LYP with basis set optimizations followed by the HF\% modification, and (d) WC1LYP with HF\% modification followed by basis set optimizations. 
The DOS (Figure 6) profiles of the atomic-orbitals components also showed profiles that were very similar, as expected. The selected bands indicate that the upper valence band is predominantly formed from oxygen $2 p_{x}, 2 p_{y}$ and $2 p_{z}$ atomic orbitals. However, the lower conduction band is predominantly formed by $\mathrm{O}$ and Mo states. The first is because of the oxygen $2 p_{x}, 2 p_{y}$, and $2 p_{z}$ atomic orbitals, and the second is the result of the $4 d_{x y}, 4 d_{x^{2}-y^{2}}$, and $4 d_{z^{2}}$ atomic orbitals.

This analysis shows that, independently of the adopted path, the final DOS structures are practically the same, as would be expected, because the structural and electronic properties are similar. It is also interesting that the change of the Fermi level of the same functional with different paths was $0.03 \%$, and those between the different functionals with the same path were $0.13 \%$, showing once again that, even if different paths are chosen, the final results are similar.

\subsection{Vibrational properties}

Besides the study of the electronic structure, a detailed study of the vibrational models, which is an important way to evaluate the reliability of the calculations since it depends on structural and electronic characteristics, was performed.

Considering the group theory, the $\mathrm{BaMoO}_{4}$ structure has 26 zone-center phonon modes: $\Gamma=$ $3 \mathrm{~A}_{\mathrm{g}}+5 \mathrm{E}_{\mathrm{g}}+5 \mathrm{~B}_{\mathrm{g}}+5 \mathrm{~A}_{\mathrm{u}}+5 \mathrm{E}_{\mathrm{u}}+3 \mathrm{~B}_{\mathrm{u}}$, where $3 \mathrm{~B}_{\mathrm{u}}$ are silent modes and $1 \mathrm{~A}_{\mathrm{u}}+1 \mathrm{E}_{\mathrm{u}}$ are acoustic modes. The active optical modes are $3 \mathrm{~A}_{\mathrm{g}}+5 \mathrm{E}_{\mathrm{g}}+5 \mathrm{~B}_{\mathrm{g}}$ Raman-active and $4 \mathrm{~A}_{\mathrm{u}}+4 \mathrm{E}_{\mathrm{u}}$ as IR-active. Table 3 lists the expected number of the active modes indicated by the group theory, as well as the experimental modes and theoretical results obtained by means of lattice dynamics calculation (LDC) according to studies found in the literature for comparison. 
According to the results shown in Table 3, the computational simulations describe the Raman modes with appreciable accuracy if compared with the experimental observations. In addition, the DFT calculations provide higher precision than the lattice dynamical calculations reported by Jindal et al..$^{51}$

Table 3. Theoretical and experimental Raman $\left(A_{g}, B_{g}\right.$, and $\left.E_{g}\right)$ and IR $\left(A_{u}\right.$ and $\left.E_{u}\right)$ active modes for the $\mathrm{BaMoO}_{4}$. Frequencies are given in $\mathrm{cm}^{-1}$.

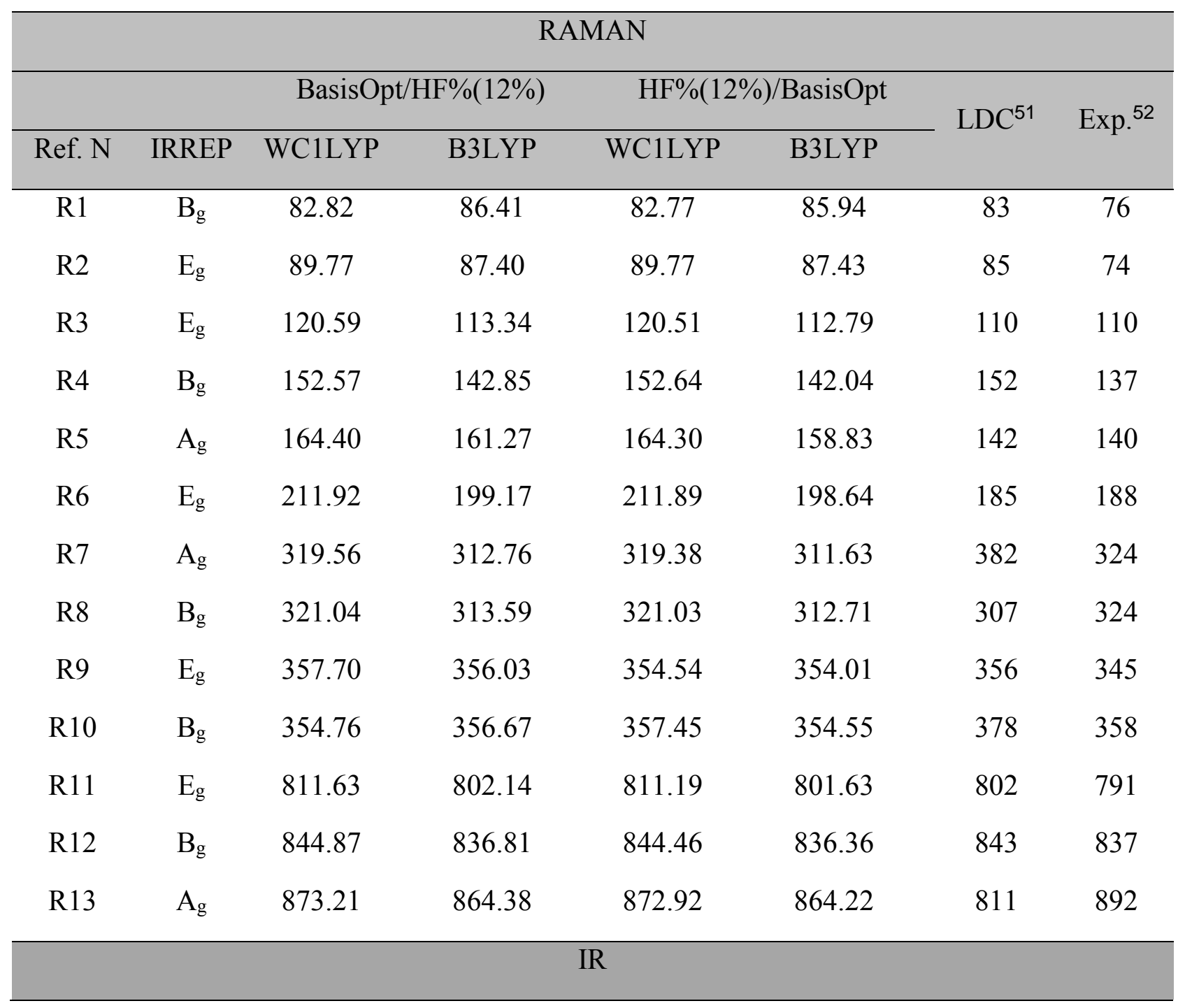




\begin{tabular}{|c|c|c|c|c|c|c|c|}
\hline \multicolumn{4}{|c|}{ BasisOpt/ HF\% (12\%) } & \multicolumn{2}{|c|}{ HF\% (12\%)/BasisOpt } & \multirow{2}{*}{ Theo. } & \multirow{2}{*}{ Exp. $^{5}$} \\
\hline Ref. N & IRREP & WC1LYP & B3LYP & WC1LYP & B3LYP & & \\
\hline IR1 & $\mathrm{E}_{\mathrm{u}}$ & 115.24 & 112.90 & 115.26 & 112.35 & - & 100 \\
\hline IR2 & $\mathrm{A}_{u}$ & 151.37 & 139.84 & 151.61 & 139.65 & - & 146 \\
\hline IR3 & $\mathrm{E}_{\mathrm{u}}$ & 164.91 & 154.23 & 164.84 & 153.50 & - & - \\
\hline IR4 & $\mathrm{A}_{u}$ & 271.92 & 276.20 & 271.63 & 274.86 & - & 290 \\
\hline IR5 & $\mathrm{E}_{\mathrm{u}}$ & 308.76 & 305.39 & 308.57 & 304.01 & - & 325 \\
\hline IR6 & $\mathrm{A}_{\mathrm{u}}$ & 376.81 & 371.92 & 376.77 & 370.27 & - & 375 \\
\hline IR7 & $\mathrm{A}_{\mathrm{u}}$ & 806.09 & 798.24 & 805.70 & 797.48 & - & 817 \\
\hline IR8 & $\mathrm{E}_{\mathrm{u}}$ & 820.96 & 811.77 & 820.48 & 811.15 & - & 881 \\
\hline
\end{tabular}

An earlier study based on DFT calculations,${ }^{37}$ showed some considerable divergences, mainly for R6 $\left(\mathrm{E}_{\mathrm{g}}\right)$, R7 $\left(\mathrm{A}_{\mathrm{g}}\right)$, and R8 $\left(\mathrm{B}_{\mathrm{g}}\right)$, with frequencies of 308,329 , and $423 \mathrm{~cm}^{-1}$, respectively. However, the systematic study of the HF\% modification and basis set optimization resulted in values very close to those of the experimental sample.

The theoretical IR modes for both functionals are also in accordance with the experimental results reported by Xiao et al. ${ }^{53}$ The third mode $\left(\mathrm{E}_{\mathrm{u}}\right)$ was not visually observed in the cited experimental study, but it is in accordance with the number of active modes in the group theory. The percentage of variation of each functional depends on each mode, but all of them showed a low deviation of the experiment.

Figure 7 depicts the atomic displacement patterns for all the Raman modes in $\mathrm{BaMoO}_{4}$. The dynamic motion can also be found in the supplementary files. 

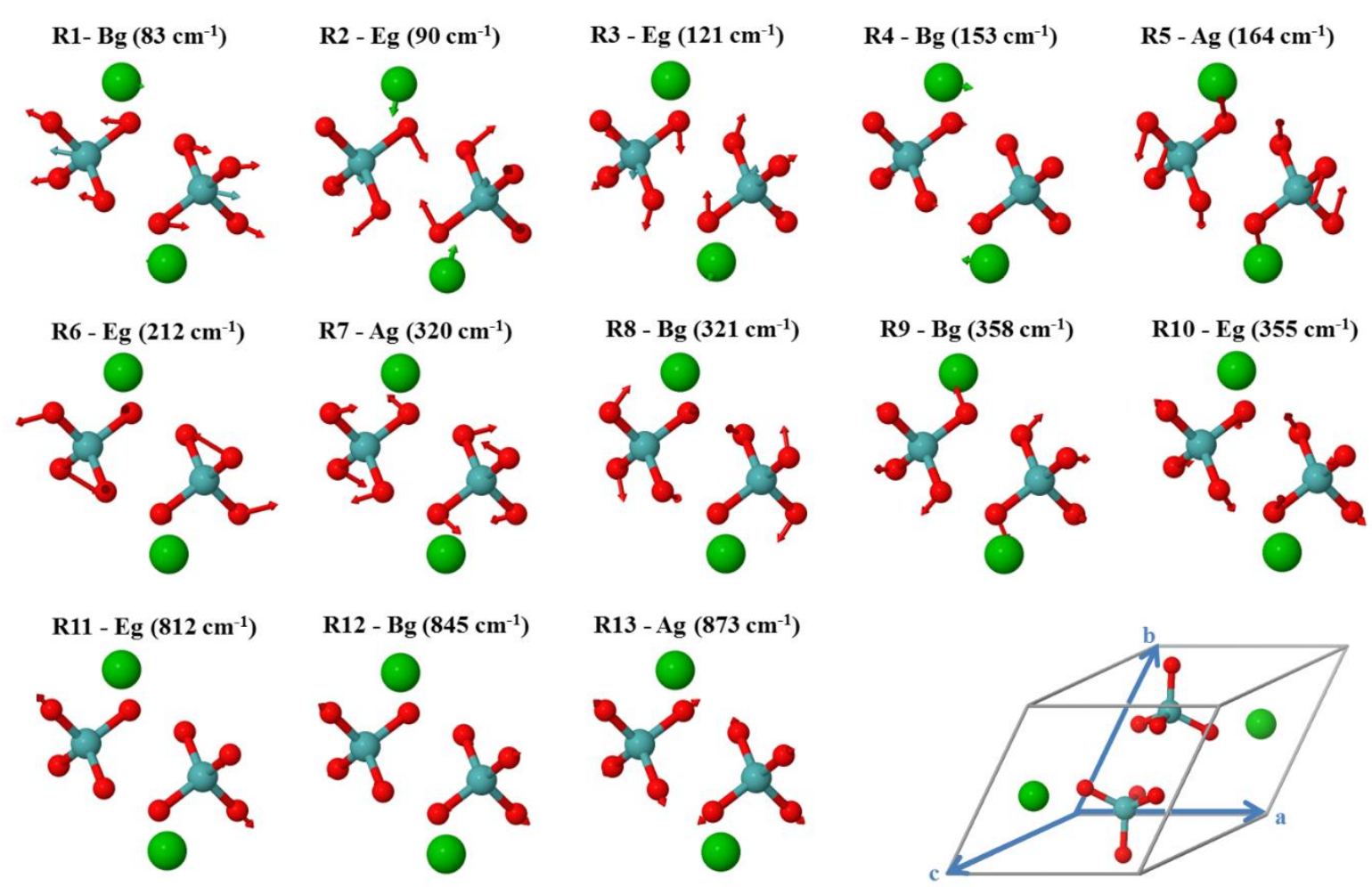

Figure 7. Schematic representation of the Raman active modes with calculated frequency right above. The amplitude of the vibrations is represented by the red arrow length. The green, blue, and red spheres denote barium, molybdenum, and oxygen atoms, respectively.

For the $\mathrm{Ag}$-like modes (Figure 7), the $\mathrm{Ba}$ and Mo atoms of the cell are fixed and the motions are attributed to the oxygen atoms. The three modes, R5, R7, and R13, are preferentially related to symmetric bending, bending and stretching, respectively, in respect to the $\mathrm{C}_{4}$ point group. In addition, these motions inside of the $\mathrm{MoO}_{4}^{-2}$ tetrahedra, with no change in the center of mass of the cluster, classify these modes within the class of internal modes.

For the five $\mathrm{B}_{\mathrm{g}}$-like modes, it is possible to divide them into two classes. $\mathrm{R} 8$, R10, and $\mathrm{R} 12$ present fixed $\mathrm{Ba}$ atoms and the motions of the $\mathrm{O}$ and Mo, with the motion, attributed almost entirely to the $\mathrm{O}$ atoms. These three modes, $\mathrm{R} 8, \mathrm{R} 10$, and $\mathrm{R} 12$, are preferentially related to antisymmetric bending, bending and stretching, respectively, with respect to the $\mathrm{C}_{4}$. However, 
they belong to the internal modes class. On the other hand, R1 and R4 show the motion of the $\mathrm{Ba}$ atoms in addition to the $\mathrm{MoO}_{4}^{-2}$ clusters, which moves like nearly rigid tetrahedrons. This class of motion is commonly known as external modes.

The five remaining motions are Eu-like modes. R2 and R3 are external modes that differ only in the referential motions of the rigid $\mathrm{MoO}_{4}{ }^{-2}$ tetrahedrons and $\mathrm{Ba}$ motion. As can be seen in Figure 7, the R2 motion presents a more pronounced rotation of the tetrahedron in relation to the Ba. The R6, R9, and R11 are included as internal motion with the Ba cation in a quasi-static. R6 and R9 are stretching modes with the movement of the all $\mathrm{O}$ atoms of the tetrahedron in directions out of a plane and with different intensities. R11 are a simple stretching vibration of one $\mathrm{O}$ atom of the $\mathrm{MoO}_{4}{ }^{-2}$ cluster. All these motions clearly broke the symmetry with respect to $\mathrm{C}_{4}$ as expected for $\mathrm{E}_{\mathrm{g}}$ modes.

In addition, a prediction of Raman and IR spectra (absorbance) were also elaborated, as shown in Figures $8 \mathrm{a}$ and $8 \mathrm{~b}$, respectively.

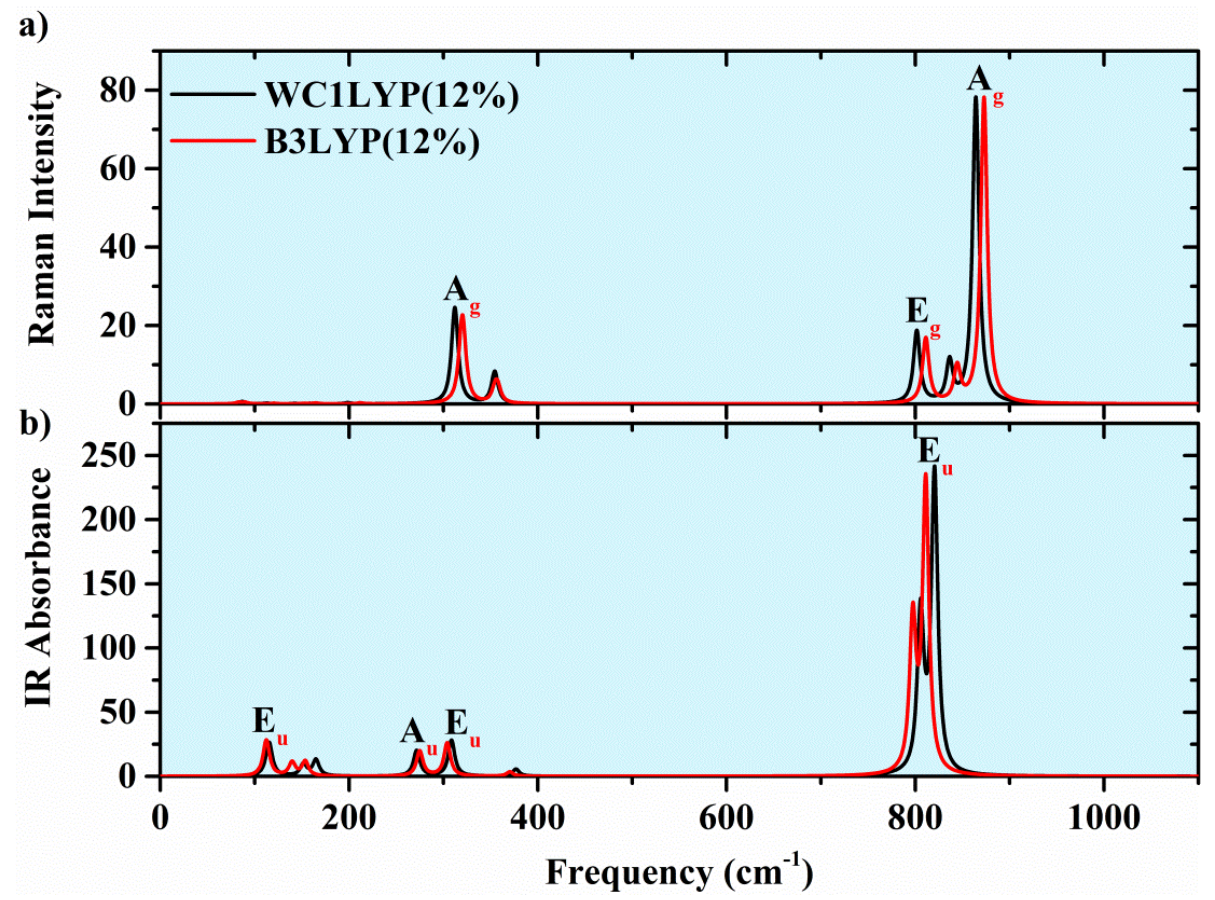

Figure 8. Theoretical first-order (a) Raman spectrum and (b) IR spectrum of $\mathrm{BaMoO}_{4}$. 
The simulated Raman spectra (Figure 8a) are similar to the experimental spectrum reported by Basiev. ${ }^{52}$ The most apparent differences are the higher intensity of the R3 mode $\left(\sim 100 \mathrm{~cm}^{-1}\right)$ and the separation of the R9 and R10 $\left(\sim 350 \mathrm{~cm}^{-1}\right)$ modes in two separated bands in comparison with the experimental results. The similarity also can be observed in the reflectivity spectrum (Figure 9) when compared with the experimental spectra reported by Xiao. ${ }^{53}$ The Raman and IR theoretical spectra are a robust indication of the ability of well-elaborated models to support experimental observations.

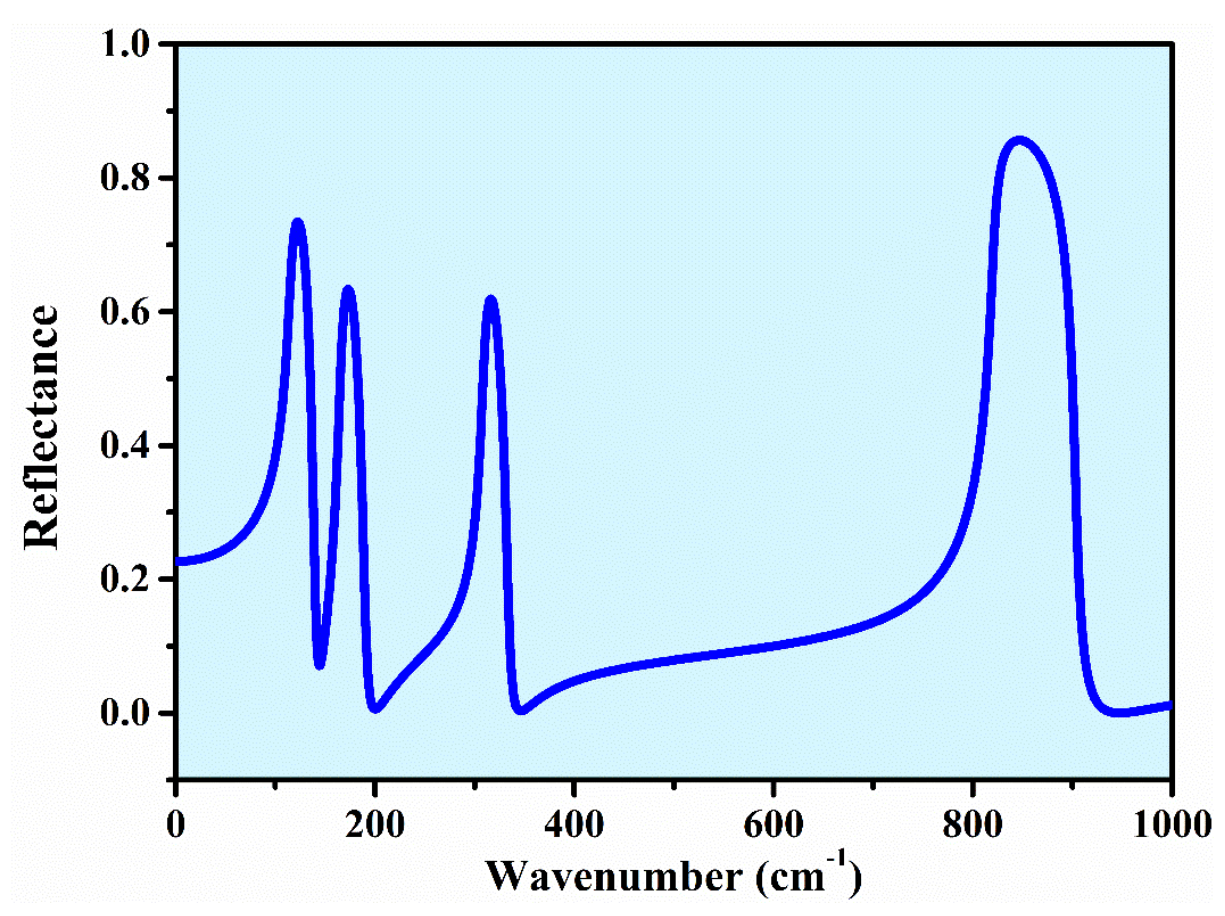

Figure 9. Theoretical reflectance of the first-order IR spectrum of $\mathrm{BaMoO}_{4}$.

In view of the result, all the models can describe the system with good precision, where the method shows slightly better results. According to the present study, a flowchart was elaborated containing systematic steps for the development of computational models for solid materials, as can be seen in Figure 10. It is believed that these steps can be used to elaborate models with a 
higher degree of reliability when compared directly with experimental systems or to extract unknown properties from an experimental point of view; thus, they can be the starting point for studies with dopant addition or structural defects.

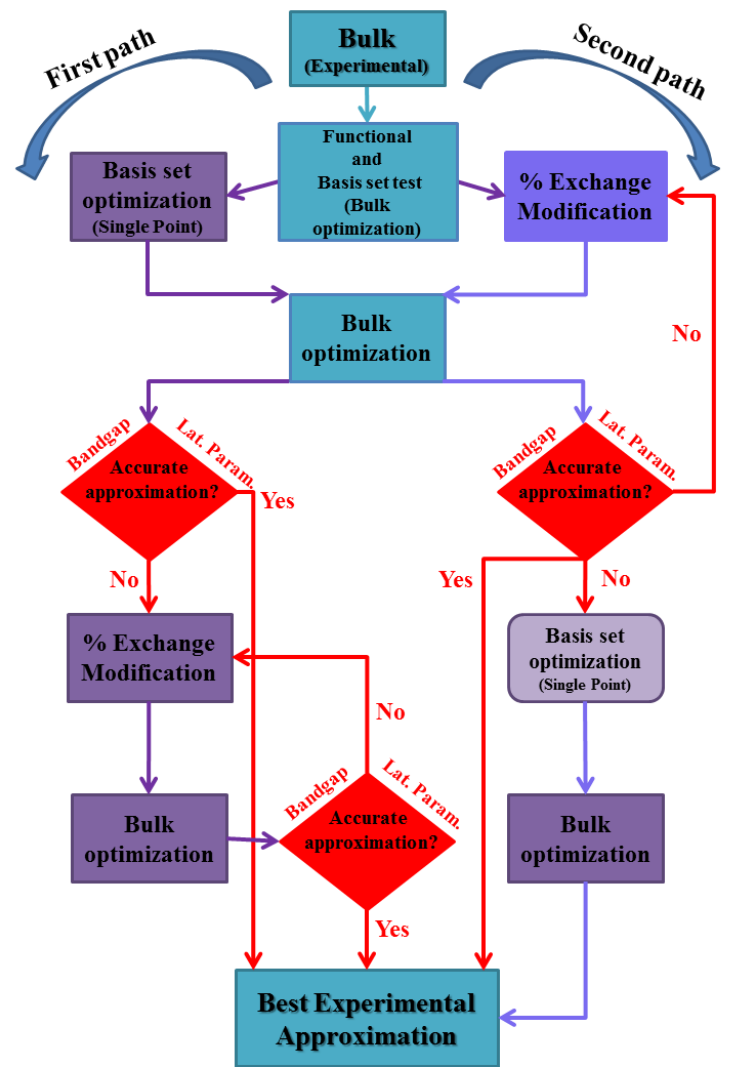

\author{
After verifying the best combination of basis set \\ and functional for the material, the procedure \\ can be done in two different paths: \\ First path: \\ 1. A basis set optimization is done to choose the \\ best coefficients or exponents in function of the \\ total energy; \\ 2. New structural optimization; \\ 3. Compare the structural and electronic properties \\ with the experimental data; \\ 4. If it is accurate, this is the final structure; \\ 5. Otherwise, modify the $\mathrm{HF} \%$; \\ 6. Reoptimize the structure; \\ 7. If it is an accurate approximation, this is the \\ final structure; \\ 8. Otherwise, return to step 5.
}

Second path:

1. Modify the Fock exchange percentage;

2. New structural optimization;

3. Compare the structural and electronic properties with the experimental data;

4. If it is accurate, this is the final structure;

5. Otherwise, return to step 1 and modify the $\mathrm{HF} \%$;

6. Then, do a basis set optimization to choose the best coefficients or exponents in function of the total energy;

7. Reoptimize the structure;

8. This is the final structure.

Figure 10. Steps for the elaboration of theoretical models with a higher level of accuracy

The first path proposed starts with a basis set optimization changing coefficients or exponents in a function of the total energy, showing agreement with the experimental or literature data. If the structure is good enough, it can be the final structure; otherwise, it is advisable to do an HF exchange modification and find the best percentage that describes the structure.

The second path proposed starts with the inverse of the first one. Initially, the HF exchange modification is done, and it is verified whether the structural and electronic properties are well 
described. If they are good enough, it can be the final structure; otherwise, a basis set optimization should be done, and then the structure is final.

\section{Conclusion}

Periodic DFT simulations were done using eight different hybrid functionals together with an all-electron basis set. The development of the best approach to describe accurately the structural, electronic and vibrational properties of a target system was proposed in two ways: 1) basis set optimizations and, when necessary, the HF\% adjustment and 2) $\mathrm{HF} \%$ adjustment followed by a base set optimization, when necessary.

Both strategies were applied to a BMO probe system, and the description, regardless of the path chosen, of the results is quite the same, with a precision of structural parameters, band gap energy, and vibrational spectra never before presented in theoretical studies of the respective materials.

The adjustment of the HF exchange functional rate should be done with caution, because the abrupt change in this percentage can result in an anomalous description of the electronic structure or perhaps other properties.

Furthermore, both pathways represented by the flowchart may help other researchers in more accurate calculations. It is also believed that the improvements generated by this methodology consist of an important step to a better description of the 2D and 1D systems because these models are usually based on the bulk results. In addition, this procedure can be applied independently of the applied package program. 


\section{ASSOCIATED CONTENT}

The following files are available free of charge.

1. Initial optimized lattice parameters and band gap energy of each tested functional (TABLE S1, PDF)

2. Optimized lattice parameters and band gap energy according to HF\% variation of both strategies. (TABLE S2 and S3, PDF)

3. Original atomic centers basis set and position of each optimized exponents. (PDF)

4. Atomic displacement motion patterns for all the Raman modes in $\mathrm{BaMoO}_{4}$. (MP4 file)

\section{AUTHOR INFORMATION}

\section{Corresponding Author}

*E-mail: sambrano@fc.unesp.br

\section{Notes}

The authors declare no competing financial interest 


\section{ACKNOWLEDGMENT}

This work was supported by the Brazilian funding agencies CNPq (432242/2018-0), CAPES (787027/2013 and 8881.068492/2014-01), and FAPESP (2013/07296-2, 2016/23891-6 and 2016/07476-9). E.O. Gomes acknowledges Generalitat Valencia for the Santiago Grisolia program (2018/064). The computational facilities were supported by resources supplied by the Molecular Simulations Laboratory, São Paulo State University, Bauru, Brazil.

\section{REFERENCES}

(1) Whitesides, G. M. Reinventing Chemistry. Angewandte Chemie International Edition 2015, 54 (11), 3196-3209.

(2) Ferrer, M. M.; Rodrigues, J. E. F. S.; Almeida, M. A. P.; Moura, F.; Longo, E.; Pizani, P. S.; Sambrano, J. R. Theoretical methods for calculations of optical phonons in BiOBr: Analysis and correction of propagated errors. J. Raman Spectros. 2018, 49 (8), 1356-1363.

(3) Jain, A.; Shin, Y.; Persson, K. A. Computational predictions of energy materials using density functional theory. Nat. Ver. Mater. 2016, 1, 15004.

(4) André, J. M.; Gouverneur, L.; Leroy, E. G. L'Etude Théorique des Systèmes Périodiques. I. La Méthode LCAO-HCO. Int. J. Quantum Chem. 1967, 1 (4), 427-450.

(5) André, J. M.; Gouverneur, L.; Leroy, E. G. L'Étude Théorique des Systèmes Périodiques. II. La Méthode LCAO-SCF-CO. Int. J. Quantum Chem. 1967, 1 (4), 451-461.

(6) Euwema, R. N.; Wilhite, D. L.; Surratt, G. T. General Crystalline Hartree-Fock Formalism: Diamond Results. Phys. Rev. B 1973, 7 (2), 818-831. 
(7) Hohenberg, P.; Kohn, W. Phys. Rev. Inhomogeneous Electron Gas. Phys. Rev. 1964, 136, B864.

(8) Kohn, W.; Sham, L. S. Self-Consistent Equations Including Exchange and Correlation Effects. Phys. Rev. 1965, 140, A1133.

(9) Fabris, G. S. L.; Marana, N. L.; Longo, E.; Sambrano, J. R. Theoretical study of porous surfaces derived from graphene and boron nitride. J. Solid State Chem. 2018, 258, 247-255.

(10) Piskunov, S.; Heifets, E.; Eglitis, R. I.; Borstel, G., Bulk properties and electronic structure of $\mathrm{SrTiO}_{3}, \mathrm{BaTiO}_{3}, \mathrm{PbTiO}_{3}$ perovskites: an ab initio HF/DFT study. Comput. Mater. Sci. 2004, 29 (2), 165-178.

(11) Cordeiro, J. M. M.; Azevedo, D. H. M.; Barretto, T. C. M.; Sambrano, J. R. Conducting Behavior of Crystalline a-PbO2 as Revealed by DFT Calculations. Mat. Res. 2018, 21, e20170641.

(12) Eglitis, R. I. Ab initio hybrid DFT calculations of $\mathrm{BaTiO}_{3}, \mathrm{PbTiO}_{3}, \mathrm{SrZrO}_{3}$ and $\mathrm{PbZrO}_{3}$ (111) surfaces. Appl. Surf. Sci. 2015, 358 (Part B), 556-562.

(13) Marana, N. L.; Casassa, S.; Longo, E.; Sambrano, J. R. Structural, Electronic, Vibrational, and Topological Analysis of Single-Walled Zinc Oxide Nanotubes. J. Phys. Chem. C 2016, 120 (12), 6814-6823.

(14) Mino, L.; Spoto, G.; Ferrari, A. M., CO2 Capture by $\mathrm{TiO}_{2}$ Anatase Surfaces: A Combined DFT and FTIR Study. J. Phys. Chem. C 2014, 118 (43), 25016-25026.

(15) Becke, A. D. Density-functional thermochemistry. III. The role of exact exchange. J. Chem. Phys. 1993, 98, 5648. 
(16) Lee, C.; Yang, W.; Parr, R. G. Development of the Colle-Salvetti correlation-energy formula into a functional of the electron density. Phys. Rev. B 1988, 37, 785.

(17) Stephens, P.; Devlin, F.; Chabalowski, C.; Frisch, M. J. Ab initio calculation of vibrational absorption and circular dichroism spectra using density functional force fields. $J$. Phys. Chem. 1994, 98 (45), 11623-11627.

(18) Bredow, T.; Gerson, A. R. Effect of exchange and correlation on bulk properties of MgO, NiO, and CoO. Phys. Rev. B 2000, 61 (8), 5194-5201.

(19) Du, P.; Yu, J. S. Photoluminescence and cathodoluminescence properties of $\mathrm{Eu}^{3+}$ ions activated $\mathrm{AMoO}_{4}(\mathrm{~A}=\mathrm{Mg}, \mathrm{Ca}, \mathrm{Sr}, \mathrm{Ba})$ phosphors. Mater. Res. Bull. 2015, 70 (Supplement C), 553-558.

(20) Rahimi-Nasrabadi, M. Strontium molybdate nanostructures: synthesis of different shapes through a new approach and its photocatalyst application. J. Mater. Sci.: Mater. Electron. 2017, 28 (2), 2200-2205.

(21) Qu, W.; Wlodarski, W.; Meyer, J. U. Comparative study on micromorphology and humidity sensitive properties of thin-film and thick-film humidity sensors based on semiconducting $\mathrm{MnWO}_{4}$. Sensor Actuat. B-Chem. 2000, 64 (1), 76-82.

(22) Yu, S. H.; Liu, B.; Mo, M. S.; Huang, J. H.; Liu, X. M.; Qian, Y. T. General Synthesis of Single-Crystal Tungstate Nanorods/Nanowires: A Facile, Low-Temperature Solution Approach. Adv. Funct. Mater. 2003, 13 (8), 639-647. 
(23) Wu, X.; Du, J.; Li, H.; Zhang, M.; Xi, B.; Fan, H.; Zhu, Y.; Qian, Y. Aqueous mineralization process to synthesize uniform shuttle-like $\mathrm{BaMoO}_{4}$ microcrystals at room temperature. J. Solid State Chem. 2007, 180 (11), 3288-3295.

(24) Sun, Y.; Ma, J.; Fang, J.; Gao, C.; Liu, Z. Synthesis of $\mathrm{BaMoO}_{4}$ high photoluminescent whiskers by an electrochemical method. Ceram. Int. 2011, 37 (2), 683-686.

(25) Adhikari, R.; Choi, J.; Narro-García, R.; De la Rosa, E.; Sekino, T.; Lee, S. W. Understanding the infrared to visible upconversion luminescence properties of $\mathrm{Er}^{3+} / \mathrm{Yb}^{3+}$ codoped $\mathrm{BaMoO}_{4}$ nanocrystals. J. Solid State Chem. 2014, 216, 36-41.

(26) Ma, X.; Zhao, W.; Wu, J.; Jia, X. Preparation of flower-like $\mathrm{BaMoO}_{4}$ and application in rechargeable lithium and sodium ion batteries. Mater. Lett. 2017, 188 (Supplement C), 248-251.

(27) Alencar, L. D. S.; Mesquita, A.; Feitosa, C. A. C.; Balzer, R.; Probst, L. F. D.; Batalha, D. C.; Rosmaninho, M. G.; Fajardo, H. V.; Bernardi, M. I. B. Preparation, characterization and catalytic application of Barium molybdate $\left(\mathrm{BaMoO}_{4}\right)$ and Barium tungstate $\left(\mathrm{BaWO}_{4}\right)$ in the gasphase oxidation of toluene. Ceram. Int. 2017, 43 (5), 4462-4469.

(28) Shivakumara, C.; Saraf, R.; Behera, S.; Dhananjaya, N.; Nagabhushana, H. Synthesis of $\mathrm{Eu}^{3+}$-activated $\mathrm{BaMoO}_{4}$ phosphors and their Judd-Ofelt analysis: Applications in lasers and white LEDs. Spectrochim. Acta A 2015, 151 (Supplement C), 141-148.

(29) Kurosaki, K.; Oyama, T.; Muta, H.; Uno, M.; Yamanaka, S. Thermoelectric properties of perovskite type barium molybdate. J. Alloy. Compd. 2004, 372 (1), 65-69.

(30) Luo, Z.; Li, H.; Shu, H.; Wang, K.; Xia, J.; Yan, Y. Synthesis of $\mathrm{BaMoO}_{4}$ nestlike nanostructures under a new growth mechanism. Cryst. Growth Des. 2008, 8 (7), 2275-2281. 
(31) Li, M.; Guan, Y.; Yin, Y.; Cui, X.; Rong, S.; Jin, G.; Hao, Y.; Wu, Q. Controllable synthesis of $3 \mathrm{D}^{\mathrm{BaXO}} 4(\mathrm{X}=\mathrm{W}, \mathrm{Mo})$ microstructures by adjusting nucleation stage and their photoluminescence properties. Superlattices Microstruct. 2015, 80 (Supplement C), 222-228.

(32) Bazarganipour, M. Synthesis and characterization of $\mathrm{BaMoO}_{4}$ nanostructures prepared via a simple sonochemical method and their degradation ability of methylene blue. Ceram. Int. 2016, $42(11), 12617-12622$.

(33) Xia, C. T.; Fuenzalida, V. M. Room temperature electrochemical growth of polycrystalline $\mathrm{BaMoO}_{4}$ films. J. Eur. Ceram. Soc. 2003, 23 (3), 519-525.

(34) Ryu, J. H.; Yoon, J. W.; Lim, C. S.; Shim, K. B. Microwave-assisted synthesis of barium molybdate by a citrate complex method and oriented aggregation. Mater. Res. Bull. 2005, 40 (9), 1468-1476.

(35) Zhao, H.; Zhang, F.; Guo, X.; Zhang, Q.; Liu, T. Ab initio study of electronic structures of $\mathrm{BaMoO}_{4}$ crystals containing an interstitial oxygen atom. J. Phys. Chem. of Solids 2010, 71 (12), 1639-1643.

(36) Qin, T.; Wang, Q.; Wang, L.; Yan, H.; Liu, C.; Han, Y.; Ma, Y.; Gao, C. High-pressure dielectric behavior of $\mathrm{BaMoO}_{4}$ : a combined experimental and theoretical study. Phys. Chem. Chem. Phys. 2016, 18 (48), 33109-33114.

(37) Sczancoski, J. C.; Cavalcante, L. S.; Marana, N. L.; da Silva, R. O.; Tranquilin, R. L.; Joya, M. R.; Pizani, P. S.; Varela, J. A.; Sambrano, J. R.; Siu Li, M.; Longo, E.; Andrés, J. Electronic structure and optical properties of $\mathrm{BaMoO}_{4}$ powders. Curr. Appl. Phys. 2010, 10 (2), 614-624. 
(38) Dovesi, R.; Erba, A.; Orlando, R.; Zicovich-Wilson, C. M.; Civalleri, B.; Maschio, L.; Rérat, M.; Casassa, S.; Baima, J.; Salustro, S.; Kirtman, B. Quantum-mechanical condensed matter simulations with CRYSTAL. WIREs. Comput. Mol. Sci. 2018, 8 (4), e1360.

(39) Santiago, A. A. G.; Almeida, C. R. R.; Tranquilin, R. L.; Nascimento, R. M.; Paskocimas, C. A.; Longo, E.; Motta, F. V.; Bomio, M. R. D. Photoluminescent properties of the $\mathrm{Ba}_{1-x} \mathrm{Zn}_{\mathrm{x}} \mathrm{MoO}_{4}$ heterostructure obtained by ultrasonic spray pyrolysis. Ceram. Int. 2018, 44 (4), 3775-3786.

(40) Rabuffetti, F. A.; Culver, S. P.; Suescun, L.; Brutchey, R. L. Structural Disorder in $\mathrm{AMoO}_{4}(\mathrm{~A}=\mathrm{Ca}, \mathrm{Sr}, \mathrm{Ba})$ Scheelite Nanocrystals. Inorg. Chem. 2014, 53 (2), 1056-1061.

(41) Bilc, D. I.; Orlando, R.; Shaltaf, R.; Rignanese, G. M.; Íñiguez, J.; Ghosez, P. Hybrid exchange-correlation functional for accurate prediction of the electronic and structural properties of ferroelectric oxides. Phys. Rev. B 2008, 77 (16), 165107.

(42) Adamo, C.; Barone, V. Toward reliable density functional methods without adjustable parameters: The PBE0 model. J. Chem. Phys. 1999, 110 (13), 6158-6170.

(43) Perdew, J. P.; Ruzsinszky, A.; Csonka, G. I.; Vydrov, O. A.; Scuseria, G. E.; Constantin, L. A.; Zhou, X.; Burke, K. Restoring the Density-Gradient Expansion for Exchange in Solids and Surfaces. Phys. Rev. Lett. 2008, 100 (13), 136406.

(44) Zhao, Y.; Truhlar, D. G. Construction of a generalized gradient approximation by restoring the density-gradient expansion and enforcing a tight Lieb-Oxford bound. J. Chem. Phys. 2008, 128 (18), 184109. 
(45) Krukau, A. V.; Vydrov, O. A.; Izmaylov, A. F.; Scuseria, G. E. Influence of the exchange screening parameter on the performance of screened hybrid functionals. J. Chem. Phys. 2006, $125(22), 224106$.

(46) Demichelis, R.; Civalleri, B.; Ferrabone, M.; Dovesi, R. On the performance of eleven DFT functionals in the description of the vibrational properties of aluminosilicates. Int. $J$. Quantum Chem. 2010, 110 (2), 406-415.

(47) Powell, M. J. D. A fast algorithm for nonlinearly constrained optimization calculations. Watson G.A. Numerical analysis, 1978, 630, 144-157.

(48) Maschio, L.; Kirtman, B.; Orlando, R.; Rèrat, M. Ab initio analytical infrared intensities for periodic systems through a coupled perturbed Hartree-Fock/Kohn-Sham method. J. Chem. Phys. 2012, 137 (20), 204113.

(49) Maschio, L.; Kirtman, B.; Rérat, M.; Orlando, R.; Dovesi, R. Ab initio analytical Raman intensities for periodic systems through a coupled perturbed Hartree-Fock/Kohn-Sham method in an atomic orbital basis. I. Theory. J. Chem. Phys. 2013, 139 (16), 164101.

(50) Maul, J.; dos Santos, I. M. G.; Sambrano, J. R.; Casassa, S.; Erba, A. A quantummechanical investigation of oxygen vacancies and copper doping in the orthorhombic $\mathrm{CaSnO}_{3}$ perovskite. Phys. Chem. Chem. Phys. 2018, 20 (32), 20970-20980.

(51) Jindal, R.; Gupta, H. C.; Sinha, M. M. A normal coordinate analysis of $\mathrm{AMoO}_{4}$ crystals $(\mathrm{A}=\mathrm{Sr}, \mathrm{Ba}$ and $\mathrm{Pb})$. Philos. Mag. 2014, 94 (2), 208-220. 
(52) Basiev, T. T.; Sobol, A. A.; Voronko, Y. K.; Zverev, P. G. Spontaneous Raman spectroscopy of tungstate and molybdate crystals for Raman lasers. Opt. Mater. 2000, 15 (3), 205-216.

(53) Xiao, E. C.; Li, J.; Wang, J.; Xing, C.; Guo, M.; Qiao, H.; Wang, Q.; Qi, Z. M.; Dou, G.; Shi, F. Phonon characteristics and dielectric properties of $\mathrm{BaMoO}_{4}$ ceramic. J. Materiomics 2018, 4 (4), 383-389. 